\title{
Numerical simulation of pararotor dynamics: Effect of mass displacement from blade plane
}

\author{
Joaquín Piechocki ${ }^{\mathrm{a}, *, 1}$, Vicente Nadal Mora ${ }^{\mathrm{a}, 1}$, Ángel Sanz-Andrés ${ }^{\mathrm{b}, 2}$ \\ a Universidad Nacional de La Plata, (1900) La Plata, Buenos Aires, Argentina \\ ${ }^{\mathrm{b}}$ Universidad Politécnica de Madrid, E-28040, Madrid, Spain
}

\section{A R T I C L E I N F O}

\section{Article history:}

Received 1 November 2013

Received in revised form 24 November 2015

Accepted 3 April 2016

Available online 21 April 2016

\section{Keywords:}

Decelerator

Numerical simulation

Rotary wing

\begin{abstract}
A B S T R A C T
The pararotor is a biology-inspired decelerator device based on the autorotation of a rotary wing, whose main purpose is to guide a load descent into a certain planetary atmosphere. This paper focuses on a practical approach to the general dynamic stability of a pararotor whose center of mass is displaced from the blade plane. The numerical simulation tool developed is based upon the motion equations of pararotor flight, utilizing a number of simplifying hypotheses that allow the most influencing factors on flight behavior to be determined. Several simulated cases are analyzed to study the effect of different parameters associated with the pararotor configuration on flight dynamics, particularly the center of mass displacement from the blade plane. It was confirmed that the ability to reach stability conditions depends mainly on a limited number of parameters associated with the pararotor configuration: the relationship between principal moments of inertia, the planform shape (associated with blade aerodynamic coefficients and blade area) and the vertical distance between the center of mass and the blade plane. As a result different types of equilibrium solutions are found and the effect of each parameter is characterized. A bifurcation in the stability shape to a precessing conical rotation, not previously found in the linear stability analysis, is predicted by this numerical model.
\end{abstract}

(c) 2016 Elsevier Masson SAS. All rights reserved.

\section{Introduction}

The pararotor is a biomimetic rotary wings decelerator, unpowered, and potentially deployable whose main practical use is to aerodynamically decelerate a load descending into a certain planetary atmosphere or to perform measurements during descent. Such a probe offers several advantages over other recovery techniques: simplicity, controlled deceleration, maneuvering capabilities and potential land recovery. Also, this decelerator type is of interest, for instance, for the measurement of atmospheric conditions around airports for aviation operations support, or the exploration of planetary atmospheres.

Works concerning the deceleration and control of falling bodies were published by Shpund and Levin [1-4], in the area of rotating parachutes. Karlsen, Borgström and Paulsson [5] worked on winged bodies for submunition applications. They reported on the

\footnotetext{
* Corresponding author.

E-mail address: joaquin.piechocki@ing.unlp.edu.ar (J. Piechocki).

1 Assistant Professor, Grupo de Ingeniería Aplicada a la Industria, Departamento de Aeronáutica, Facultad de Ingeniería, Calle 116 entre 47 y 48.

2 Full Professor, Instituto Universitario de Microgravedad "Ignacio Da Riva," Escuela Técnica Superior de Ingenieros Aeronáuticos, Plaza Cardenal Cisneros, 3.
}

advantages of the pararotor over the parachute: lower sensitivity to lateral winds, parachute deployment problems, lower precession movements, and higher falling velocity.

The flight of samara wings has similarities with pararotors. Seter and Rosen [6,7] studied numerically the influence of different parameters on samara flight stability. Crimi [8] has studied a rotating body with only one wing for submunition applications. He searched for a body that performed periodic movements.

Previous work has been carried out concerning modeling the stability of a pararotor, mainly by Nadal Mora, Sanz-Andres and Piechocki [9-17]. They conducted investigations concerning the stability behavior of pararotors whose blades where aligned with the center of mass of the whole device. They developed an analytical model that predicts the dynamic behavior under different device configurations.

An analytical linearized model was described by Piechocki et al. [17] presenting four different cases of analyses that are revisited in the current work.

Seter and Rosen [18] presented the modeling of multibody systems for a helicopter.

Pararotor decelerator potential has been investigated by different authors [19-22], who consider applications that are centered 


\section{Nomenclature}

$C_{D} \quad$ drag coefficient of the blade

$C_{L \alpha} \quad$ slope of the curve lift vs. angle of attack of the blade

$C_{D M} \quad$ model drag coefficient

$C_{M} \quad$ center of mass

$C_{P} \quad$ center of pressure

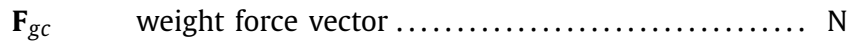

h kinetic momentum ................. kg m/s $\mathrm{s}^{2}$

$I_{i} \quad$ principal moments of inertia, $i=1,2,3 \ldots \ldots \mathrm{kg} \mathrm{m}^{2}$

$k \quad$ vertical velocity to blade-tip-speed ratio

$k_{i j} \quad$ ratio of coordinates of the center of pressure of the blade $j$ in axis $i$ direction, $r_{i j} / r_{11}$

$k_{e} \quad$ dimensionless parameter used for the stability analysis

$\mathbf{n}_{i} \quad$ direction perpendicular to blade $i$ surface, $i=1,2$

$S \quad$ area of one blade ........................ $\mathrm{m}^{2}$
$\mathbf{T}_{\mathrm{M}}$ transformation matrix to convert vectors from the body fixed frame to the inertial frame

$\mathbf{V}_{r i} \quad$ relative velocity to the $i$ blade $\ldots \ldots \ldots \ldots \ldots \ldots . \ldots \ldots . \mathrm{m} / \mathrm{s}$

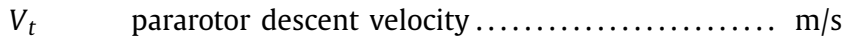

$v_{h} \quad$ axial hovering velocity $\ldots \ldots \ldots \ldots \ldots \ldots \ldots \ldots \ldots \ldots \ldots \ldots \ldots, \mathrm{m}$

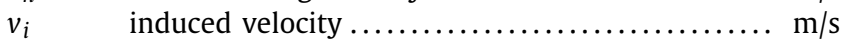

$\alpha \quad$ angle of attack.......................... rad

$\beta_{0}, \beta_{1}, \beta_{2}$ mean pitch angle, pitch angle of blade 1,2 ,

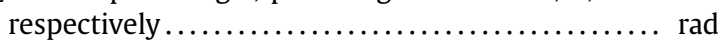

$\theta \quad$ nutation angle $\ldots \ldots \ldots \ldots \ldots \ldots \ldots \ldots \ldots \ldots \ldots \ldots, \ldots \ldots \ldots$

$\varphi \quad$ spin angle $\ldots \ldots \ldots \ldots \ldots \ldots \ldots \ldots \ldots \ldots \ldots \ldots, \ldots \ldots, \ldots \ldots d$

$\psi \quad$ precession angle $\ldots \ldots \ldots \ldots \ldots \ldots \ldots \ldots \ldots \ldots \ldots, \ldots \ldots \ldots$ rad

$\omega \quad$ angular velocity $\ldots \ldots \ldots \ldots \ldots \ldots \ldots \ldots \ldots \ldots, \mathrm{rad} / \mathrm{s}$

$\omega_{12}$ angular velocity projected in the 1-2 plane, or

transversal angular velocity ................. $\mathrm{rad} / \mathrm{s}$ in mission concepts requiring controlled descent, low-velocity landing, and atmospheric research capability on planet exploration.

The main objective of the current work is to develop and validate a numerical simulation tool to describe pararotor flight modes to determine the stability behavior of a pararotor considering different configuration parameters, particularly the effect of the distance between the blade plane and the center of mass (illustrated in Fig. 1) and to study dynamic effects of parameters associated with the device configuration.

It is worth mentioning that the present paper extends the studies previously developed [17] considering nonlinear effects in the pararotors dynamics and their results on pararotor flight behavior. Those nonlinear terms are the result of using Newton-Euler equations for a rigid body excited with aerodynamic loads coupled with inertial phenomena. In the analytical model [17] second order terms were consistently neglected by considering that the following parameters are of small magnitude: blade pitch angle, relationship between descent rate to the velocity induced in the blades by rotation, distance of blade center of pressure in $\mathbf{e}_{2}$ direction from center of mass, blade drag aerodynamic coefficient. The nature of Newton-Euler equations of a rigid body under the effect of aerodynamic forces (that are blade relative velocity dependent) is in general nonlinear. The validity of the linear analytical model [17] is restricted to the vicinity of an equilibrium point. This numerical approach is a useful potential tool that allows the stability limits, the effects of configurational parameters, and the flight mode of future practical devices to be more accurately defined. This modelization includes neither simplificative assumptions (magnitude orders neglecting criteria) nor linearization of the system near the equilibrium solutions, as developed in [17]. The significance of this work is that the nonlinear factors effects on flight dynamics can be considered practically, not only in terms of response time, but also in stability limits and flight mode, particularly with pararotors flying near their stability limits defined in [17].

The current research shows solutions that cannot be reached by previous modelization, and so, a deeper understanding of the phenomena can be reached, with its positive practical impact on pararotor design.

\section{Mathematical and numerical models}

The approach chosen in this work to study the pararotor dynamic behavior was to build an analytical model of a pararotor based on the development of the complete motion equations, including aerodynamic forces and torques generated by the blades and to compute numerically the evolution of the system from a particular initial flight attitude, different from the final equilibrium state. So, the analyses performed responds to the observation of patterns over specific cases. The effect of $k_{31}$, the dimensionless distance of the center of mass to the blade plane in the falling direction, is analyzed together with a number of geometrical and aerodynamical parameters.

Induced velocity, $v_{i}$, was not directly included in the model, following similar assumptions of former studies [16]. Other studies [21] indicated that real low aspect ratio rotary wing shows an induced power correction factor (that can be defined as the factor that modifies rotor classic momentum theory when considering nonideal blade physical effects), $\kappa$, of about 2 or greater. This fact indicates that the relationship of descent rate and axial hovering velocity, $v_{h}$, is greater than 2 , thus in a windmill brake state axial descent [22]. The current model focuses on the assumption of small induced velocity, in the windmill brake state. Large induced power correction factors associated with low aspect ratio wing are consistent with this hypothesis. Vertical tunnel tests performed [20] using different small aspect ratio wings pararotors showed induced velocities of $15 \%$ of the descent velocity on average, evidencing that the simplificative hypothesis is associated with a representative flight range.

Flow model for axial descent in windmill brake state [22] indicates that the sensibility of induced velocity, $v_{i}$, to descent rate, decreases with the descent velocity to the hovering velocity ratio, $V_{t} / v_{h}$. This means that vertical velocity disturbances influence on induced velocity depends on the $v_{i} / v_{h}$ magnitude, and so on $\kappa$.

However, the effect of induced velocity is included in the values of lift, lift slope and drag coefficients, $C_{L}, C_{L \alpha}$ and $C_{D}$, which are determined from experiments [16]. This experimental work was performed in operational conditions of a pararotor model, so the effect of $v_{i}$ will result in an increment of the magnitudes of lift and drag if free stream velocity over the blades is considered. As a consequence, the model will be developed associated with this experimental data, that will define an operating range of validity, over which the lift slope can be approximated as linear. This range is defined in the incompressible regime.

The system analyzed is a pararotor flying in an autorotation regime, modeled as an inertial cylindrical body with two identical low-aspect-ratio flat blades which rotates at angular velocity $\omega$ and falls vertically at speed $V_{t}$. The geometry is defined in Figs. 1 and 2 . The body-fixed reference frame, $1,2,3$, has its origin at the center of mass and directions $\mathbf{e}_{1}, \mathbf{e}_{2}, \mathbf{e}_{3}$. The axes $1,2,3$ are the principal axes of the body. The inertial reference frame is $X, Y, Z$; its axes have the directions $\mathbf{i}, \mathbf{j}, \mathbf{k}$. The blades are located on a plane parallel to the plane 1,2 , in the direction $\mathrm{P}_{1} \mathrm{P}_{2}$. 


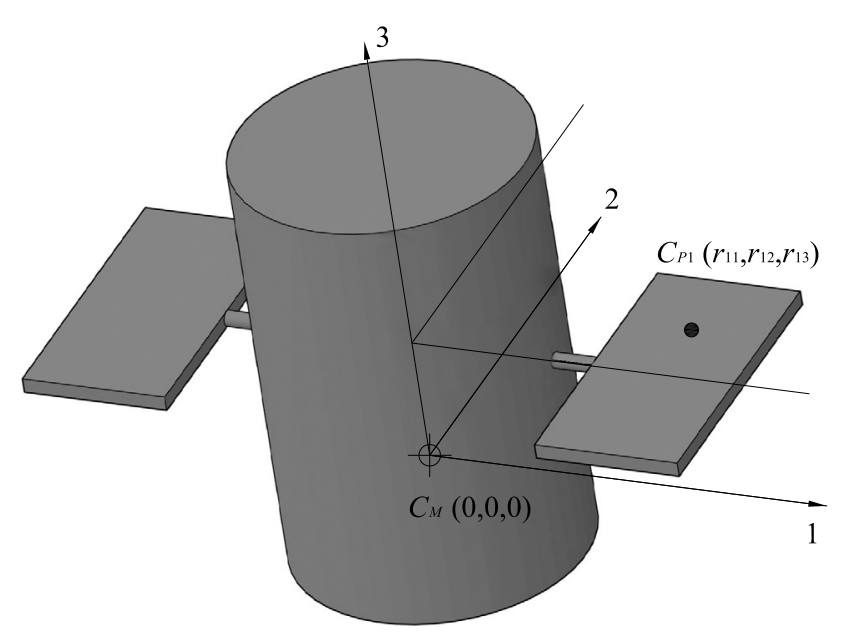

Fig. 1. Scheme of the studied pararotor. Position of the center of mass, $C_{M}$, and center of pressure, $C_{P 1}$, in body axes.

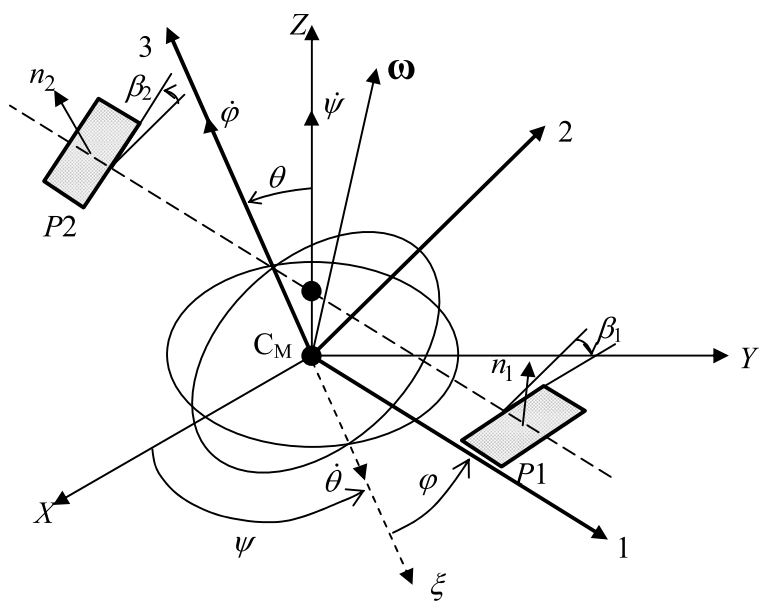

Fig. 2. System geometry. $(X, Y, Z)$, inertial reference frame; $(1,2,3)$, body-fixed reference frame; $\psi$, precession angle; $\theta$, nutation angle; $\varphi$, spin angle; $\omega$, angular velocity; $\beta_{1}, \beta_{2}$, blade $\mathrm{P}_{1}$ and $\mathrm{P}_{2}$ pitch angles, respectively; $\zeta$, auxiliary axis; $\mathrm{B}$, intersection of blade plane with axis 3 .

To develop the mathematical model used for the numerical simulations it was necessary to: state a representation system that allows migration from a body fixed coordinate frame to inertial coordinate frame and vice versa, set the complete dynamics equations for the pararotor, set the expressions for forces and moments acting on the pararotor, including aerodynamic and gravity forces, considering the distance from the center of mass to the blade plane, and state the numerical computation order and data-flow.

The tool adopted to develop the numerical simulation is an open source software called Scicos. To study the pararotor dynamic response by numerical simulation a time interval and initial conditions were established.

\subsection{Pararotor orientation}

A very important issue for the development of a pararotor flight dynamics simulator is to track the spatial position and attitude of the pararotor. This fact implies having the capability to express vectors from the body fixed coordinate system in the inertial system, and the other way around, in a univocal way, avoiding singularities along the numerical process.

A normalized quaternion is used to express the pararotor orientation. Therefore, the relative attitude of the pararotor in the inertial coordinate system is expressed in terms of four parameters (or a vector plus a scalar).

The use of quaternions to express pararotor attitude implies setting a rotation matrix that allows coordinates and vectors to be transformed from one reference system to the other, in agreement to the expressions summarized by Diebel [23].

To numerically compute the orientation over a period of time, the derivative of the orientation quaternion with respect to time, $t$, is integrated from the initial conditions (given by an initial orientation quaternion, equivalent to the orientation described by initial Euler angles) in differential steps. As a result, an orientation quaternion corresponding to each integration step over time is obtained, which will be the initial orientation quaternion for the next time step. The arithmetic operations over the quaternion introduce a cumulative distortion on the orthonormality of the element. The algebraic method introduced by Ai Chun Fang and Zimmerman [25] was used to reduce this effect. The method introduces a dimensionless constant $K_{\varepsilon}$ called normalization gain. It is demonstrated that the method is stable if $h K_{\varepsilon}<1 \mathrm{~s}$ (by the direct method of Lyapunov), where $h$ is the time step. So the values adopted after testing the performance over different numerical examples are $K_{\varepsilon}=1$, and $h<1 / 10 \mathrm{~s}$.

\subsection{Initial conditions}

The initial orientation conditions for the numerical simulation are defined by a set of Euler angles $\mathbf{E}_{u l 0}=\left(\varphi_{0}, \theta_{0}, \psi_{0}\right)$, which corresponds to an initial orientation quaternion. After each time step, the simulation tool outputs an orientation quaternion, corresponding to a set of Euler angles. To facilitate the spatial representation, these Euler angles are expressed in the so-called 3-1-3 order [24]: rotation is described by a rotation along 3 -axis, a sequential rotation along 1-axis, and a final rotation along 3-axis.

\subsection{Dynamics model}

The complete pararotor dynamics system has six degrees of freedom, and includes coupled rotations and translations. The expressions, in the principal inertia axes body reference frame, are:

$\mathbf{M}=\mathbf{I} \cdot \mathrm{d} \boldsymbol{\omega} / \mathrm{d} t+\boldsymbol{\omega} \times(\mathbf{I} \cdot \boldsymbol{\omega})$,

$\mathbf{F}=m\left(\mathrm{~d} \mathbf{V}_{b} / \mathrm{d} t+\omega \times \mathbf{V}_{b}\right)$,

where $\boldsymbol{\omega}=\left[\omega_{1}, \omega_{2}, \omega_{3}\right]$ is the rotational speed vector, $\mathbf{V}_{b}=$ $\left[V_{b 1}, V_{b 2}, V_{b 3}\right]$ is the center of gravity velocity vector, $\mathbf{M}=$ $\left[M_{1}, M_{2}, M_{3}\right]$ is the momentum vector, $\mathbf{F}=\left[F_{1}, F_{2}, F_{3}\right]$ is the thrust vector, $\mathbf{I}$ is the principal moments of inertia tensor and $m$ is the mass of the pararotor.

The numerical simulation is based on the integration of the dynamics equations, starting from initial values for angular acceleration and translational acceleration. The integration process is sequenced so that it results in an angular velocity, a translational velocity and a position at every time step. The simulation parameters considered are: integration time, numerical integration method, time step, initial conditions (orientation, position, speed).

The relative velocity of the flow with regard to the blades 1 and 2 , on a quiet atmosphere, is given by:

$\mathbf{V}_{r 1}=-\boldsymbol{\omega} \times \mathbf{r}_{1}-\mathbf{V}_{b}, \quad \mathbf{V}_{r 2}=-\boldsymbol{\omega} \times \mathbf{r}_{2}-\mathbf{V}_{b}$

where $\mathbf{r}_{1}=\left[r_{11}, r_{12}, r_{13}\right]^{T}$ and $\mathbf{r}_{2}=\left[r_{21}, r_{22}, r_{23}\right]^{T}$ are the vector positions of the center of pressure of the blades 1 and 2 .

Normalized vectors having the lift $\left(\mathbf{e}_{L}\right)$ and $\operatorname{drag}\left(\mathbf{e}_{D}\right)$ directions are considered for each blade, as can be seen in Fig. 3. Induced velocity is associated with blade aerodynamic coefficients determination. 


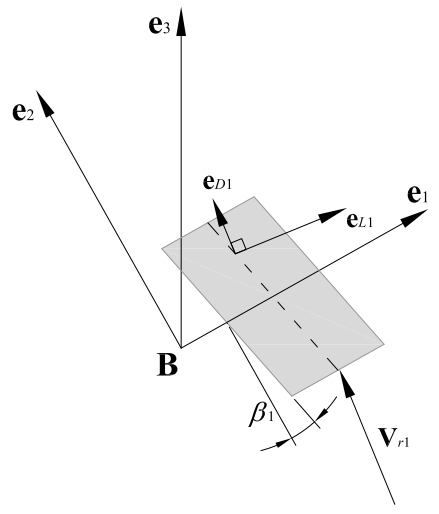

Fig. 3. Representation of normalized vectors $\mathbf{e}_{D 1}, \mathbf{e}_{L 1}$ in the directions of the lift and drag of the $\mathrm{P}_{1}$ blade. $\mathbf{e}_{1}, \mathbf{e}_{2}, \mathbf{e}_{3}$, are parallel to the axes $1,2,3 ; \beta_{1}$, blade pitch angle for blade $1 ; \mathbf{V}_{r 1}$ flow velocity relative to blade 1 .

The normalized lift vector, $\mathbf{e}_{L i}$, is normal to the plane defined by the relative speed, $\mathbf{V}_{r 1}$, and the axis along the span direction of the blade, $\mathbf{e}_{1}$. The component of the aerodynamic load along axis $\mathbf{e}_{1}$ is not considered because aerodynamic forces on the blades in this direction are neglected as a hypothesis. The relative air speed to each blade may have a component along $\mathbf{e}_{1}$ when a lateral pararotor displacements appear. This component is, in general, considerably smaller than the others, and appears with a cyclic behavior around a null average magnitude. The impact of neglecting aerodynamic load along $\mathbf{e}_{1}$ on the model was tested, initially appearing to validate the hypothesis at first look as its effects didn't show a remarkable contribution to dynamics. The impact of this second order effect may be subject of further research. The normalized drag vector, $\mathbf{e}_{D 1}$, has the direction of the relative velocity of each blade.

The angles of attack, $\alpha_{1}, \alpha_{2}$, are given by the following equations:

$\sin \alpha_{1}=\mathbf{n}_{1} \mathbf{V}_{r 1} / \operatorname{norm}\left(\mathbf{V}_{r 1}\right), \quad \sin \alpha_{2}=\mathbf{n}_{2} \mathbf{V}_{r 2} / \operatorname{norm}\left(\mathbf{V}_{r 2}\right)$,

where $\mathbf{n}_{1}=\left[0, \sin \beta_{1}, \cos \beta_{1}\right], \mathbf{n}_{2}=\left[0,-\sin \beta_{2}, \cos \beta_{2}\right]$, are unit vectors normal to blade 1,2 respectively.

The aerodynamic forces of lift, $\mathbf{L}_{i}$, and $\operatorname{drag}, \mathbf{D}_{i}$, and moments, $\mathbf{M}_{i}$, generated by the blades, as well as the gravity force generated at the center of mass, are the input elements that act over the rigid body dynamics. The aerodynamic forces are easily described in a body centered reference frame, and the gravity force in an inertial reference frame. The used global aerodynamic coefficients of the blades include the effects of the induced velocity. These aerodynamic coefficients could be adjusted to match experimental results with the theoretical model. As a reference in the aerodynamic coefficient determination, previous work [15] has been considered.

The aerodynamic coefficient is determined experimentally. These coefficients are linearized around a pararotor operating a steady state.

This fact implies defining an operating range of validity of the aerodynamic coefficients over which the lift slope can be approximated as linear.

These moments and forces are given by:

$$
\begin{aligned}
& \mathbf{L}_{i}=\frac{1}{2} \rho S C_{L \alpha} \alpha_{i}\left|\mathbf{V}_{r i}\right|^{2} \mathbf{e}_{L i}, \\
& \mathbf{D}_{i}=\frac{1}{2} \rho S C_{D}\left|\mathbf{V}_{r i}\right|^{2} \mathbf{e}_{D i}, \\
& \mathbf{F}_{g c}=\mathbf{T}_{\mathrm{M}}[0,0,-m g], \\
& \mathbf{M}=\left[\mathbf{r}_{1} \times \mathbf{L}_{1}+\mathbf{r}_{1} \times \mathbf{D}_{1}+\mathbf{r}_{2} \times \mathbf{L}_{2}+\mathbf{r}_{2} \times \mathbf{D}_{2}\right] .
\end{aligned}
$$

Table 1

Physical characteristics of the model [19].

\begin{tabular}{ll}
\hline Parameter & Value \\
\hline Mass, kg & 0.31 \\
Blade chord, m & 0.74 \\
Blade span, m & 0.15 \\
$I_{1}, \mathrm{~kg} \mathrm{~m}^{2}$ & $2.229 \cdot 10^{-4}$ \\
$I_{2}, \mathrm{~kg} \mathrm{~m}^{2}$ & $9.930 \cdot 10^{-3}$ \\
$I_{3}, \mathrm{~kg} \mathrm{~m}^{2}$ & $1.010 \cdot 10^{-3}$ \\
$I_{21} ; I_{12}, \mathrm{~kg} \mathrm{~m}^{2}$ & $-5.860 \cdot 10^{-5}$ \\
$I_{13} ; I_{31}, \mathrm{~kg} \mathrm{~m}^{2}$ & $5.690 \cdot 10^{-7}$ \\
$I_{23} ; I_{32}, \mathrm{~kg} \mathrm{~m}^{2}$ & $-1.170 \cdot 10^{-5}$ \\
\hline
\end{tabular}

Table 2

Mean measured performance of samara tests. Comparison with numerical results.

\begin{tabular}{lccc}
\hline Performance parameter & Free flight [18] & Numerical simulation & Error \\
\hline$\omega_{3}, \mathrm{rad} / \mathrm{s}$ & 17.1 & 17.1 & $0.1 \%$ \\
$V_{t}, \mathrm{~m} / \mathrm{s}$ & 2.8 & 2.8 & $0.1 \%$ \\
$\theta, \mathrm{rad}$ & 0.52 & 0.48 & $7.6 \%$ \\
\hline
\end{tabular}

\subsection{Integration method}

An integration method is used to integrate the completely developed equation system (1) and (4)-(7) with respect to flight time. Different methods were compared in practical simulations to determine the solution error magnitude. In particular, the RungeKutta non-adaptive iterative methods, studied by Butcher [26], were analyzed and tested. Finally a Dormand-Prince method (5th order) was chosen, together with a time integration step of $0.005 \mathrm{~s}$, as a compromise between error magnitude and computational cost.

To validate the numerical simulation of the pararotor dynamics with a known solution, the free motion of a rigid body was chosen. A rigid body moving without external forces or moment action, whose main parameters are defined in Table 1, was numerically simulated and compared with the analytical solution, given in [27]. The excellent agreement between the angular velocities given by the analytical solution and the numerical simulation, showed the efficiency of the simulation tool to represent body dynamics. An error parameter to compare analytical results and numerical simulations at each integration step was defined as $E_{123}(t)=$ $\operatorname{sqrt}\left(\left(\omega_{1 \mathrm{~T}}(t)-\omega_{1 \mathrm{~N}}(t)\right)^{2}+\left(\omega_{2 \mathrm{~T}}(t)-\omega_{2 \mathrm{~N}}(t)\right)^{2}+\left(\omega_{3 \mathrm{~T}}(t)-\omega_{3 \mathrm{~N}}(t)\right)^{2}\right)$ where subscripts $\mathrm{T}$ and $\mathrm{N}$ represent the analytical and numerical results, respectively. The result of the error parameter evaluation over $60 \mathrm{~s}$ remained bounded by a magnitude of $10^{-4} \mathrm{rad} / \mathrm{s}$.

\subsection{Comparison between the numerical simulation and experimental results}

Experimental measurements over samara flights have been reported by different authors. These data can be used to compare the numerical simulation with experimental measured parameters, as a complementary validation approach that is not intended to be fully conclusive. The samara type, single bladed pararotor, free flight case taken by Kellas [19] is adopted, because it includes enough information to obtain the necessary simulation parameters.

The physical characteristics of the model are given in Table 1 .

The performance parameters used in the comparison are: spin, vertical velocity and nutation angle. Results are summarized in Table 2. They correspond to an equilibrium solution so that the constant parameter could be measured in an experiment. A good agreement can also be appreciated in this case. Differences could be due to the aerodynamic model employed.

On the other hand, a set of experiments were used to compare their results with numerical simulation as another validation approach. The experimental setup consisted of a vertical wind tun- 
Table 3

Mean measured performance of pararotor tests (C05P02). Comparison with numerical results.

\begin{tabular}{|c|c|c|c|c|c|c|c|}
\hline \multicolumn{2}{|c|}{ Pitch angles } & \multicolumn{3}{|c|}{ Experimental results } & \multicolumn{3}{|c|}{ Numerical simulation results } \\
\hline$\beta_{1}$, rad & $\beta_{2}$, rad & $\omega_{3}, \mathrm{rad} / \mathrm{s}$ & $V_{t}, \mathrm{~m} / \mathrm{s}$ & $\theta_{e q}$, rad & $\omega_{3}, \mathrm{rad} / \mathrm{s}$ & $V_{t}, \mathrm{~m} / \mathrm{s}$ & $\theta_{e q}$, rad \\
\hline-2 & -2 & 287 & 4.2 & 0 & 287 & 4.2 & 0 \\
\hline-4 & -4 & 308 & 4.3 & 0 & 296 & 4.8 & 0 \\
\hline-6 & -6 & 341 & 4.7 & 0 & 307 & 5.5 & 0 \\
\hline
\end{tabular}

Table 4

General parameters of the pararotor cases.

\begin{tabular}{ll}
\hline Parameter & Value \\
\hline$C_{L \alpha}$ & 1.35 \\
$C_{D}$ & 0.15 \\
$\rho, \mathrm{kg} / \mathrm{m}^{3}$ & 1.21 \\
$S, \mathrm{~m}^{2}$ & 0.0254 \\
$r_{11}, r_{21}, \mathrm{~m}$ & 0.037 \\
$r_{12}, r_{22}, \mathrm{~m}$ & 0.016 \\
$\beta_{1}, \beta_{2}, \mathrm{rad}$ & 0.07 \\
$\varphi_{0}, \mathrm{rad}$ & 0.00 \\
$\theta_{0}, \mathrm{rad}$ & 0.10 \\
$\psi_{0}, \mathrm{rad}$ & 0.00 \\
\hline
\end{tabular}

nel where a pararotor was tested in a stable flight condition. The experimental conditions are described by Nadal Mora et al. [28]. The tested models consisted of a hollow cylinder with two rectangular blades of bare aluminum alloy, named in [28] with the code C05P02, with $k_{31}=0$. The following parameters have been measured: the spin velocity, measured by means of a stroboscopic lamp (resolution $50 \mathrm{rpm}$ ); the blade pitch angle, with a goniometer (resolution $1 \mathrm{deg}$ ); the flow velocity by a standard pitot-tube NPL type and a micromanometer (resolution 0.5 Pa). A comparison between experiments and numerical simulation results are shown in Table 3.

In Table 3 a good fit between experiments and simulations can be observed, for the case where $k_{31}=0$ and the Straight Solution is presented. The nutation angle for the equilibrium point, $\theta_{e q}$, presents no difference between experimental results and numerical simulation. The descent rate, $V_{t}$, displays differences from $0 \%$ to $11 \%$. The spin velocity, $\omega_{3}$, exhibits differences from $0 \%$ to $15 \%$.

To have more conclusive results over the simulation approach to pararotor flight, free flight tests, or vertical tunnel tests, should be performed extensively, considering different pararotors configurations, which are being carried out. Transient response should also be contrasted with experimental results.

\section{Results of the numerical model}

In the present section four groups of cases are considered regarding the relationships between principal inertia moments: A $\left(I_{3}>I_{2}, I_{1}\right)$, B $\left(I_{3}<I, I_{1}\right) ; C\left(I_{1}>I_{3}>I_{2}\right)$ and $\mathrm{D}\left(I_{2}>I_{3}>I_{1}\right)$. The groups of cases are the same as those presented in previous studies [17]; they correspond to the pararotor configurations that can be built. Only Case A results are presented. The groups of cases were chosen in order to explore the dynamical response of the pararotor presented by Piechocki and Nadal Mora $[9,10]$. Each group includes case variants to explore the effect of the distance between the center of mass and the blade plane, and the pitch angles, on the pararotor dynamics.

Cases A and B are pararotor configurations where the principal moment of inertia $I_{3}$ is the highest and the smallest, respectively. Cases $\mathrm{C}$ and $\mathrm{D}$ are configurations where $I_{3}$ has an intermediate value between $I_{1}$ and $I_{2}$.

The physical parameters of the baseline configuration considered are presented in Table 4. This baseline configuration is set to attain possible technological interest of pararotor use. The analysis
Table 5

Principal moments of inertia.

\begin{tabular}{lrrrl}
\hline Variable & \multicolumn{1}{c}{ Case A } & \multicolumn{1}{c}{ Case B } & \multicolumn{1}{c}{ Case C } & \multicolumn{1}{l}{ Case D } \\
\hline$I_{1}, \mathrm{~kg} \mathrm{~m}^{2}$ & $5.4 \cdot 10^{-6}$ & $6.0 \cdot 10^{-6}$ & $22.0 \cdot 10^{-6}$ & $21.0 \cdot 10^{-6}$ \\
$I_{2}, \mathrm{~kg} \mathrm{~m}^{2}$ & $21.2 \cdot 10^{-6}$ & $22.0 \cdot 10^{-6}$ & $6.0 \cdot 10^{-6}$ & $23.0 \cdot 10^{-6}$ \\
$I_{3}, \mathrm{~kg} \mathrm{~m}^{2}$ & $25.9 \cdot 10^{-6}$ & $16.0 \cdot 10^{-6}$ & $16.0 \cdot 10^{-6}$ & $15.0 \cdot 10^{-6}$ \\
\hline
\end{tabular}

of the dynamics is focused mainly on the evolution of the nutation angle.

The initial conditions for the numerical simulations were given by: $\omega_{0}=\left[\omega_{10}, \omega_{20}, \omega_{30}\right]^{T}=\left[0.00 \mathrm{rad} / \mathrm{s}, 0.00 \mathrm{rad} / \mathrm{s}, \omega_{\mathrm{eq}}\right]^{T}$.

In all cases, the total duration of the numerical simulation is set in such a way that the equilibrium solution is completely established. The moments of inertia values are given in Table 5 .

\subsection{Case $A\left(I_{3}>I_{2}, I_{1}\right)$}

The present case considers the condition of $I_{3}$ being the biggest moment of inertia.

Analytical results of the simplified analytical method are presented together with the numerical simulation results. The analytical results were found using the expressions of the simplified linear analytical model developed by Piechocki, Nadal Mora and Sanz Andrés [13]. The differences between numerical simulation and analytical results are considered to be due to the higher order dynamics effects and non-linear behavior. The numerical simulation considers a number of effects neglected on the simplified analytical model, that extends the validity range and the tool strength. These neglected effects on the simplified model are originated from truncating terms in the aerodynamic forces and momentum equations, considering only the terms of highest magnitude for what was considered a general configuration [10]. Also, on the simplified analytical model, the linearization strategy followed to generate a coefficient matrix in order to evaluate the system stability implies neglecting nonlinear effects.

The first set of tests (Table 6) show that for cases where $k_{31}=0$ there is only one equilibrium solution type, characterized by a nutation angle, $\theta$, which is 0 or is very close to 0 radians (axis of rotation aligned or almost aligned with axis 3 in the body reference frame). This family of equilibrium solutions includes small angular velocities $\omega_{e q 1}, \omega_{e q 2}$ in the order of $1 \mathrm{rad} / \mathrm{s}$. For simplicity this solution is named Straight Solution, or Type 1 Solution. The results of the simulations and of the linear analytical model are also shown in Table 6. It can be noted that both ways of computing the equilibrium solutions are coherent if the equilibrium solution reached is the Straight one. In this case relative errors between variables are small, increasing as the difference between $\beta_{1}$ and $\beta_{2}$ increases (evidencing the influence of nonlinear terms). Finally, it can be noted that the analytical model tends to systematically overestimate the magnitude of the variables in comparison with the numerical simulation, when the equilibrium solution is a Straight Solution. These results are verified for simulation tests performed for $k_{31}=0.0$.

In Table 7 the existence of two equilibrium solution types is shown. One of these solutions, for example for $\beta_{2}=0.07$ (with $\beta_{1}=0.07$ ), shows a null or a very small nutation angle, and the angular velocities $\omega_{e q 1}$ and $\omega_{e q 2}$ are constant, with magnitudes 
Table 6

Case A - equilibrium results from numerical simulation and analytical model for $k_{31}=0$.

\begin{tabular}{|c|c|c|c|c|c|c|c|c|c|c|}
\hline \multirow[t]{2}{*}{ Blades pitch } & \multicolumn{5}{|c|}{ Numerical simulation } & \multicolumn{5}{|c|}{ Analytical model } \\
\hline & $\omega_{e q 1}, \mathrm{rad} / \mathrm{s}$ & $\omega_{e q 2}, \mathrm{rad} / \mathrm{s}$ & $\omega_{e q 3}, \mathrm{rad} / \mathrm{s}$ & $V_{t}, \mathrm{~m} / \mathrm{s}$ & $\theta_{e q}$, rad & $\omega_{e q 1}, \mathrm{rad} / \mathrm{s}$ & $\omega_{e q 2}, \mathrm{rad} / \mathrm{s}$ & $\omega_{e q 3}, \mathrm{rad} / \mathrm{s}$ & $V_{t}, \mathrm{~m} / \mathrm{s}$ & $\theta_{e q}$, rad \\
\hline$\beta_{1}=0.07 ; \beta_{2}=0.07$ & 0.0000 & 0.0000 & 292.8 & 4.57 & 0.000 & 0.0000 & 0.0000 & 292.8 & 4.57 & 0.000 \\
\hline$\beta_{1}=0.07 ; \beta_{2}=0.09$ & 0.0303 & 0.0581 & 294.0 & 4.66 & $2.229 \cdot 10^{-4}$ & 0.0327 & 0.0618 & 294.0 & 4.66 & $2.377 \cdot 10^{-4}$ \\
\hline$\beta_{1}=0.07 ; \beta_{2}=0.14$ & 0.1072 & 0.2056 & 297.2 & 4.88 & $7.802 \cdot 10^{-4}$ & 0.1165 & 0.2198 & 297.2 & 4.88 & $8.370 \cdot 10^{-4}$ \\
\hline$\beta_{1}=0.07 ; \beta_{2}=0.19$ & 0.1856 & 0.3561 & 300.2 & 5.11 & $1.338 \cdot 10^{-3}$ & 0.2030 & 0.3830 & 300.2 & 5.11 & $1.444 \cdot 10^{-3}$ \\
\hline$\beta_{1}=0.07 ; \beta_{2}=0.21$ & 0.2174 & 0.4170 & 301.4 & 5.21 & $1.560 \cdot 10^{-3}$ & 0.2384 & 0.4497 & 301.4 & 5.21 & $1.689 \cdot 10^{-3}$ \\
\hline$\beta_{1}=0.07 ; \beta_{2}=0.25$ & 0.2816 & 0.5403 & 303.7 & 5.41 & $2.006 \cdot 10^{-3}$ & 0.3106 & 0.5859 & 303.7 & 5.41 & $2.184 \cdot 10^{-3}$ \\
\hline
\end{tabular}

Table 7

Case A - equilibrium results from numerical simulation and analytical model $-k_{31}=0.7$.

\begin{tabular}{|c|c|c|c|c|c|c|c|c|c|c|}
\hline \multirow[t]{2}{*}{ Blades pitch } & \multicolumn{5}{|c|}{ Numerical simulation } & \multicolumn{5}{|c|}{ Analytical model } \\
\hline & $\overline{\omega_{e q 1}, \mathrm{rad} / \mathrm{s}}$ & $\omega_{e q 2}, \mathrm{rad} / \mathrm{s}$ & $\omega_{e q 3}, \mathrm{rad} / \mathrm{s}$ & $V_{t}, \mathrm{~m} / \mathrm{s}$ & $\theta_{e q}$, rad & $\omega_{e q 1}, \mathrm{rad} / \mathrm{s}$ & $\omega_{e q 2}, \mathrm{rad} / \mathrm{s}$ & $\omega_{e q 3}, \mathrm{rad} / \mathrm{s}$ & $V_{t}, \mathrm{~m} / \mathrm{s}$ & $\theta_{e q}$, rad \\
\hline $\begin{array}{l}\beta_{1}=0.07 \\
\beta_{2}=0.07\end{array}$ & 0.0000 & 0.0000 & 292.8 & 4.57 & 0.000 & 0.0000 & 0.0000 & 292.8 & 4.57 & 0.000 \\
\hline $\begin{array}{l}\beta_{1}=0.07 \\
\beta_{2}=0.09\end{array}$ & 0.0322 & 0.0184 & 294.0 & 4.66 & $1.250 \cdot 10^{-4}$ & 0.0298 & 0.0170 & 294.0 & 4.66 & $1.167 \cdot 10^{-4}$ \\
\hline $\begin{array}{l}\beta_{1}=0.07 \\
\beta_{2}=0.11\end{array}$ & 0.0650 & 0.0355 & 295.3 & 4.75 & $2.490 \cdot 10^{4}$ & 0.0599 & 0.0330 & 295.3 & 4.75 & $2.316 \cdot 10^{-4}$ \\
\hline $\begin{array}{l}\beta_{1}=0.07 \\
\beta_{2}=0.113\end{array}$ & $\begin{array}{l}0.0750^{*} \\
\left(3.025^{* *}\right)\end{array}$ & $\begin{array}{l}0.0375^{*} \\
\left(2.908^{* *}\right)\end{array}$ & $\begin{array}{l}295.0^{*} \\
\left(0.007^{* *}\right)\end{array}$ & $\begin{array}{l}4.77^{*} \\
\left(0.007^{* *}\right)\end{array}$ & $\begin{array}{l}0.117^{* *} \\
\left(0.005^{* *}\right)\end{array}$ & 0.0643 & 0.0349 & 295.0 & 4.77 & $2.481 \cdot 10^{-4}$ \\
\hline $\begin{array}{l}\beta_{1}=0.07 \\
\beta_{2}=0.12\end{array}$ & $\begin{array}{l}0.0827^{*} \\
\left(4.017^{* *}\right)\end{array}$ & $\begin{array}{l}0.0400^{*} \\
\left(3.840^{* *}\right)\end{array}$ & $\begin{array}{l}294.7^{*} \\
\left(0.010^{* *}\right)\end{array}$ & $\begin{array}{l}4.81^{*} \\
\left(0.011^{* *}\right)\end{array}$ & $\begin{array}{l}0.156^{*} \\
\left(0.007^{* *}\right)\end{array}$ & 0.0747 & 0.0396 & 294.7 & 4.81 & $2.870 \cdot 10^{-4}$ \\
\hline $\begin{array}{l}\beta_{1}=0.07 \\
\beta_{2}=0.13\end{array}$ & $\begin{array}{l}0.1000^{*} \\
\left(5.120^{* *}\right)\end{array}$ & $\begin{array}{l}0.0534^{*} \\
\left(4.917^{* *}\right)\end{array}$ & $\begin{array}{l}295.1^{*} \\
\left(0.019^{* *}\right)\end{array}$ & $\begin{array}{l}4.87^{*} \\
\left(0.021^{* *}\right)\end{array}$ & $\begin{array}{l}0.199^{*} \\
\left(0.009^{* *}\right)\end{array}$ & 0.0898 & 0.0463 & 295.1 & 4.87 & $3.423 \cdot 10^{-4}$ \\
\hline $\begin{array}{l}\beta_{1}=0.07 \\
\beta_{2}=0.14\end{array}$ & $\begin{array}{l}0.1000^{*} \\
\left(6.100^{* *}\right)\end{array}$ & $\begin{array}{l}0.0650^{*} \\
\left(5.825^{* *}\right)\end{array}$ & $\begin{array}{l}295.2^{*} \\
\left(0.073^{* *}\right)\end{array}$ & $\begin{array}{l}4.93^{*} \\
\left(0.0315^{* *}\right)\end{array}$ & $\begin{array}{l}0.236^{*} \\
\left(0.010^{* *}\right)\end{array}$ & 0.1048 & 0.0524 & 295.2 & 4.93 & $3.968 \cdot 10^{-4}$ \\
\hline $\begin{array}{l}\beta_{1}=0.07 \\
\beta_{2}=0.19\end{array}$ & $\begin{array}{l}0.2250^{*} \\
\left(9.875^{* *}\right)\end{array}$ & $\begin{array}{l}0.1075^{*} \\
\left(9.463^{* *}\right)\end{array}$ & $\begin{array}{l}295.0^{*} \\
\left(0.075^{* *}\right)\end{array}$ & $\begin{array}{l}5.21^{*} \\
\left(0.081^{* *}\right)\end{array}$ & $\begin{array}{l}0.385^{*} \\
\left(0.017^{* *}\right)\end{array}$ & 0.1793 & 0.0753 & 295.0 & 5.21 & $6.593 \cdot 10^{-4}$ \\
\hline $\begin{array}{l}\beta_{1}=0.07 \\
\beta_{2}=0.21\end{array}$ & $\begin{array}{l}0.2500^{*} \\
\left(11.150^{* *}\right)\end{array}$ & $\begin{array}{l}0.1200^{*} \\
\left(10.710^{* *}\right)\end{array}$ & $\begin{array}{l}294.7^{*} \\
\left(0.095^{* *}\right)\end{array}$ & $\begin{array}{l}5.33^{*} \\
\left(0.105^{* *}\right)\end{array}$ & $\begin{array}{l}0.437^{*} \\
\left(0.019^{* *}\right)\end{array}$ & 0.2089 & 0.0809 & 294.7 & 5.33 & $7.601 \cdot 10^{-4}$ \\
\hline $\begin{array}{l}\beta_{1}=0.07 \\
\beta_{2}=0.25\end{array}$ & $\begin{array}{l}0.3750^{*} \\
\left(13.730^{* *}\right)\end{array}$ & $\begin{array}{l}0.1750^{*} \\
\left(13.125^{* *}\right)\end{array}$ & $\begin{array}{l}293.9^{*} \\
\left(0.144^{* *}\right)\end{array}$ & $\begin{array}{l}5.55^{*} \\
\left(0.156^{* *}\right)\end{array}$ & $\begin{array}{l}0.539^{*} \\
\left(0.024^{* *}\right)\end{array}$ & 0.2677 & 0.0863 & 293.9 & 5.55 & $9.569 \cdot 10^{-4}$ \\
\hline
\end{tabular}

smaller than $1 \mathrm{rad} / \mathrm{s}$. This solution is qualitatively identical to that found for $k_{31}=0$, the Straight Solution. The other solution appears in Case $\mathrm{A}$ for $k_{31} \geq 0.7$ or for $k_{31} \leq-1.0$ (Table 9). This class of solution is not predicted by the analytical model, and represents a bifurcation of the solution. This solution type is characterized by: 1) nutation angles considerably larger than the previous case, 2) a sinusoidal periodic behavior of $\omega_{e q 1}, \omega_{e q 2}$ with an amplitude of considerable magnitude; and 3) a sinusoidal fluctuation of $\omega_{\text {eq }}$ showing small amplitude. This solution is hereafter named as the Conical Solution, or Type 2 Solution because the angular velocity vector, $\boldsymbol{\omega}$, moves along a cone with elliptical cross-section with small excentricity. Simulation tests for $k_{31}>0.7$ showed a strong tendency to conical solutions.

As shown in Fig. 4, the equilibrium solution of the numerical simulation shows a bifurcation from the Straight Solution at a given limit of the pitch angle, $\beta_{2}$, in the case $k_{31}=0.7$. If this limit is surpassed, the Conical Solution appears abruptly. The nutation angle, $\theta_{e q}$, for Conical Solution $\left(\theta_{e q}>5^{\circ}\right)$ is significantly larger than for Straight Solution $\left(\theta_{e q} \approx 1^{\circ}\right)$. These large angles could reduce the prediction capacity of the aerodynamics model. To substantiate this point a campaign of experimentation would be required.

For simplicity, henceforth we will call Case A the Base Case.

Table 8 summarizes the time response and equilibrium nutation angle, with different $k_{31}$ values. In these calculations, the integration time was increased until the nutation solution remained bounded between a \pm 0.1 degree range.

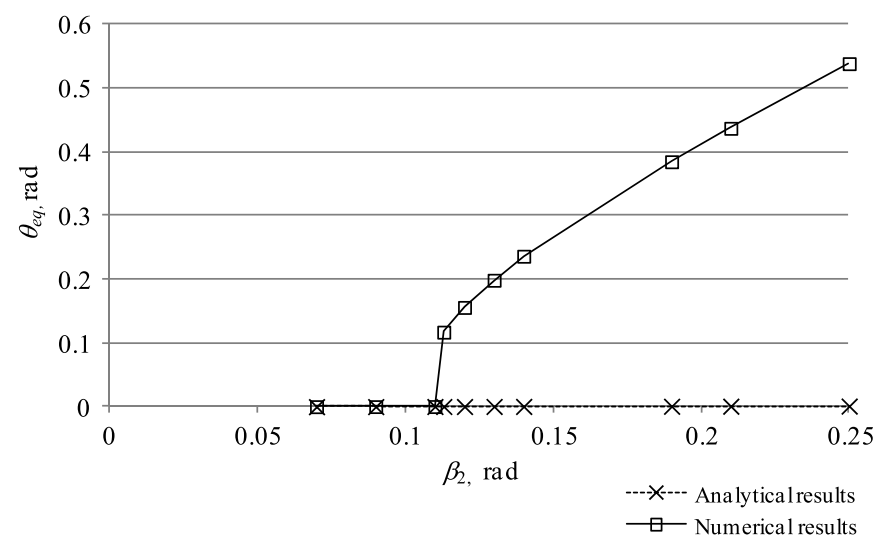

Fig. 4. Bifurcation diagram. Variation of the nutation angle at equilibrium, $\theta_{e q}$, with blade 2 pitch angle $\beta_{2}$, for $\beta_{1}=0.07$ and $k_{31}=0.7$.

The simulations performed showed that when $k_{31}=-10$, nutation angles are higher than $90 \mathrm{deg}$. This means that the pararotor turns upside down, and a stable solution (that can be considered anomalous from a practical point of view) is reached with nutation angles of considerable magnitude in that attitude, as in the Conical Solution case. This solution is denoted Type 3 Solution.

As shown in Fig. 5 and Table 8, the transient evolution of the system from the initial conditions and the equilibrium solution type are directly related to the magnitude of $k_{31}$. 
Table 8

Time needed to reach the equilibrium point, $t_{r e}$, for different pararotor cases over the Case A.

\begin{tabular}{|c|c|c|c|c|c|c|}
\hline \multirow[t]{2}{*}{ Case } & \multicolumn{2}{|c|}{$\beta_{1}=0.01, \beta_{2}=0.01$} & \multicolumn{2}{|c|}{$\beta_{1}=0.07, \beta_{2}=0.07$} & \multicolumn{2}{|c|}{$\beta_{1}=0.07, \beta_{2}=0.14$} \\
\hline & $t_{r e}, \mathrm{~s}$ & $\theta_{e}, \operatorname{deg}$ & $t_{r e}, \mathrm{~s}$ & $\theta_{e}, \operatorname{deg}$ & $t_{r e}, \mathrm{~s}$ & $\theta_{e}, \operatorname{deg}$ \\
\hline$k_{31}=100$ & 4.20 & $0.0^{\mathrm{T} 1}$ & 3.9 & $0.0^{\mathrm{T} 1}$ & 2.6 & $0.0^{\mathrm{T} 1}$ \\
\hline$k_{31}=10$ & 12.0 & $45.8^{\mathrm{T} 2}$ & 12.1 & $45.8^{\mathrm{T} 2}$ & 16.2 & $40.7^{\mathrm{T} 2}$ \\
\hline$k_{31}=1.0$ & 63.2 & $34.3^{\mathrm{T} 2}$ & 49.9 & $34.3^{\mathrm{T} 2}$ & 44.4 & $38.7^{\mathrm{T} 2}$ \\
\hline$k_{31}=0.5$ & 30.1 & $0.0^{\mathrm{T} 1}$ & 32.2 & $0.0^{\mathrm{T} 1}$ & 37.5 & $0.0^{\mathrm{T} 1}$ \\
\hline$k_{31}=0.0$ & 7.8 & $0.0^{\mathrm{T} 1}$ & 8.1 & $0.0^{\mathrm{T} 1}$ & 8.5 & $0.0^{\mathrm{T} 1}$ \\
\hline$k_{31}=-0.5$ & 6.8 & $0.0^{\mathrm{T} 1}$ & 7.9 & $0.0^{\mathrm{T} 1}$ & 9.6 & $0.0^{\mathrm{T} 1}$ \\
\hline$k_{31}=-1.0$ & 14.3 & $0.0^{\mathrm{T} 1}$ & 24.1 & $0.0^{\mathrm{T} 1}$ & 47.5 & $0.0^{\mathrm{T} 1}$ \\
\hline$k_{31}=-2.0$ & 43.5 & $39.2^{\mathrm{T} 2}$ & 28.2 & $39.2^{\mathrm{T} 2}$ & 23.5 & $45.4^{\mathrm{T} 2}$ \\
\hline$k_{31}=-10$ & 7.7 & $132.1^{\mathrm{T} 3}$ & 10.6 & $128.3^{\mathrm{T} 3}$ & 11.0 & $126.1^{\mathrm{T} 3}$ \\
\hline
\end{tabular}

Table 9

Effect on the dynamic behavior of the pararotor (time to reach equilibrium, $t_{r e}$ ) of the parameters $k_{31}, k_{21}, I_{1} / I_{3}, C_{L \alpha} / C_{D}$.

\begin{tabular}{|c|c|c|c|c|c|}
\hline$k_{31}$ & $k_{21}$ & $I_{1} / I_{3}$ & $C_{L \alpha} / C_{D}$ & Equilibrium solution & Results \\
\hline 0 & Increase & & & Type 1 & $t_{r e}$ increases. \\
\hline 0 & Decrease & & & Type 1 & $t_{r e}$ decreases. \\
\hline 0 & & Increase & & Type 1 & $t_{r e}$ increases. \\
\hline 0 & & Decrease & & Type 1 & $t_{r e}$ decreases. \\
\hline 0 & & & Increase & Type 1 & $t_{r e}$ increases. \\
\hline 0 & & & Decrease & Type 1 & $t_{r e}$ decreases. \\
\hline$>0$ & & & & Type 1 or 2 & $t_{r e}$ increases. Nutation angle increases. \\
\hline$>0$ & Increase & & & Type 1 & $t_{r e}$ decreases a second order magnitude. \\
\hline$>0$ & Increase & & & Type 2 & $t_{r e}$ increases. Nutation angle decreases. \\
\hline$>0$ & Decrease & & & Type 1 & $t_{r e}$ decreases. \\
\hline$>0$ & Decrease & & & Type 2 & $t_{r e}$ increases. Nutation angle decreases. \\
\hline$>0$ & & Increase & & Type 1 or 2 & $t_{r e}$ increases. Nutation angle increases to instability. \\
\hline$>0$ & & Decrease & & Type 1 or 2 & $t_{r e}$ decreases. \\
\hline$>0$ & & & Increase & Type 1 or 2 & $t_{r e}$ increases. Nutation angle increases to instability. \\
\hline$>0$ & & & Decrease & Type 1 or 2 & $t_{r e}$ decreases. Nutation angle decreases. \\
\hline$<0$ & & & & Type 1,2 or 3 & $t_{r e}$ increases. \\
\hline$<0$ & Increase & & & Type 1,2 or 3 & $t_{r e}$ decreases. \\
\hline$<0$ & Decrease & & & Type 1,2 or 3 & $t_{r e}$ increases to instability. \\
\hline$<0$ & & Increase & & Type 1,2 or 3 & $t_{r e}$ decreases. \\
\hline$<0$ & & Decrease & & Type 1,2 or 3 & $t_{r e}$ increases to instability. \\
\hline$<0$ & & & Increase & Type 1,2 or 3 & $t_{r e}$ increases to instability. \\
\hline$<0$ & & & Decrease & Type 1,2 or 3 & $t_{r e}$ decreases. \\
\hline
\end{tabular}

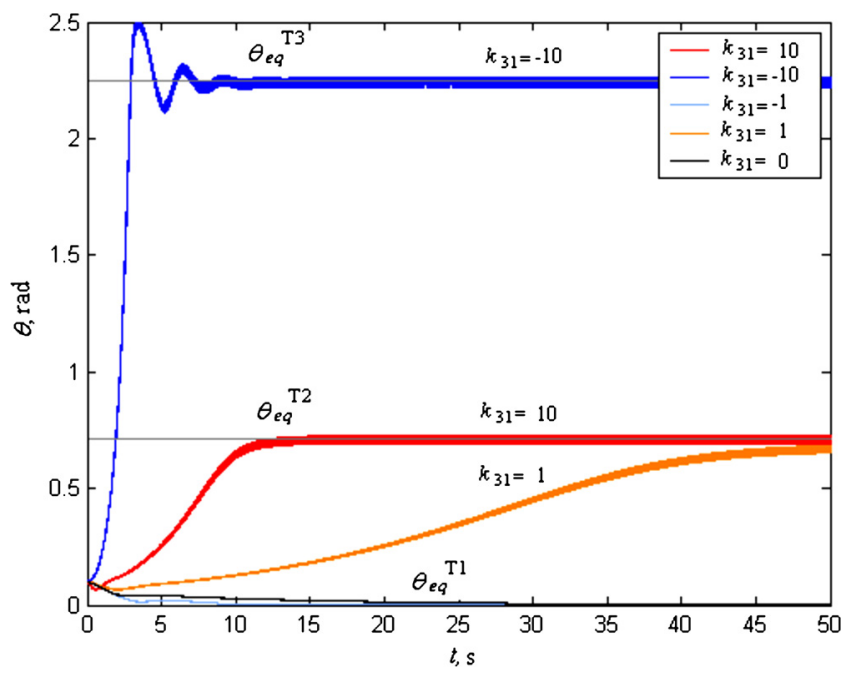

Fig. 5. Variation of the nutation angle, $\theta$, with time, $t$, for different values of the position parameter $k_{31}$. Initial nutation angle is set as $\theta_{0}=0.1 \mathrm{rad}$. Superscripts T1, T2, T3 denote the equilibrium type.

From the analyses of cases shown, the model behavior can be summarized by using a certain number of parameters, whose influence patterns are shown in Table 9.

It can be concluded that for $I_{3}>I_{2}, I_{1}$ four different solutions can be reached: Straight, Conical, Type 3 and Dynamic instability.
For $k_{31}=0$ only two possibilities exist: Straight solution or Dynamic instability.

The effect of $k_{31}$ (if different from 0 ) depends on its magnitude and sign, geometrical configuration, and aerodynamics performance. The effect can be described in terms of the stabilization time and of the solution type.

Conical solution assumes a nutation angle significantly bigger than 0 . A considerable increase in $k_{31}$ tends to diminish the sensibility of the pararotor of destabilizing factors. However, if the sign of $k_{31}$ is negative and its magnitude is significant, the solution tends to be Type 3 .

With regard to the effect of increasing $k_{21}$, for $k_{31}=0$, it implies an increase in stabilization time. If $k_{31}>0$, when the solution is Straight, there is a value of $k_{21}$ that makes the stabilization time maximum. When the solution is Conical, there is a value of $k_{21}$ that makes the stabilization time minimum. It can be seen that if $k_{31}<0$, increasing $k_{21}$ leads to a decrease in stabilization time.

Regarding $I_{1} / I_{3}$ and $I_{2} / I_{3}$ (in the case $I_{3}>I_{2}>I_{1}$ ) it can be observed that independently of the value of $k_{31}$, the influence on the dynamic response is given by the relationship between the biggest and smallest inertia moments.

The Case A simulations indicate that an increase in $I_{1} / I_{3}$ for $k_{31}=0$ implies an increase in transient time, up to instability.

Besides, the results show that an increase in $I_{1} / I_{3}$ for $k_{31}>0$ implies an increase in transient time, up to instability, going through equilibrium solutions Straight and Conical. Also, if $I_{1} / I_{3}$ diminishes, for $k_{31}>0$, then a smaller transient time appears than in the base case. 
Finally, it was also shown that an increment of $I_{1} / I_{3}$ for $k_{31}<0$ implies an increment of transient time, up to instability. Also, that if $I_{1} / I_{3}$ diminishes, an absolute minimum transient time can be found, related with the moments of inertia.

With regard to the parameter $C_{L \alpha} / C_{D}$, it was shown that independently of $k_{31}$ value, its increment implies a growth on transient time.

\section{Conclusions}

The numerical model developed to analyze the stability of the motion has been validated by two comparisons: 1) between analytical model results and numerical simulation, and 2) between experimental results and numerical simulations. Taking into account this validation approach, the numerical simulation developed can be considered to be a useful and reliable tool to describe pararotor flight. Transient behavior should be validated with a free flight test campaign.

From the observations made, it can be noted that four solution types are possible, denoted as Straight, Conical, Type 3 and Dynamic instability. From these solutions, when $k_{31}=0$, numerical simulations showed that the possibilities are reduced to solutions Straight and Dynamic instability. Therefore, it can be said that there are new phenomena derived from the existence of non-zero values of $k_{31}$ that introduce Conical and Type 3 equilibrium solutions, also denoted as conical solutions. It is worth noting that the analytical approach is able to predict accurately enough the pararotor dynamic response when $k_{31}=0$. The differences between numerical simulation and analytical approach come from the higher order dynamics effects and non-linear behavior considered in the simulation model.

This higher order dynamics effects and non-linear behavior appear when the model flights near the stability limits described in [17]. This simulation model allows for consideration of the effect of not small angles of attack, of the existence of a component along axis 1 of the relative velocity, of existence of a distance from the aerodynamic center of the blades to the center of mass in the direction perpendicular to the blade span, and of angular velocities along axis 1 and 2, $\omega_{1}$ and $\omega_{2}$, not small compared with $\omega_{3}$.

The variation of $k_{31}$ mainly affects to stability. In the case a stable configuration is given, $k_{31}$ has an impact on the type of equilibrium solution and on stabilization time. The stability is given by the ratio of principal moments of inertia as it was determined on the work above mentioned [10].

While Straight Solution is characterized by nutation angles close to zero, in Conical solution a significant nutation angle appears, configuring a conical motion of the rotation axis. For $k_{31}$ different from zero, the system shows a bifurcation from Straight Solution to Conical, depending on the pararotor configuration, the aerodynamic characteristics of the device, and $k_{31}$ magnitude.

In any case, it can be said that a significant increment of $k_{31}$ magnitude tends to reduce the sensitivity of the pararotor to destabilizing factors.

The general conclusion is that the parameters $k_{31}, C_{L \alpha} / C_{D}$, $\beta_{1}$ and $\beta_{2}$ can be used to control the dynamic behavior of the pararotor. Both, the stabilization time of the system to a given perturbation and the type of equilibrium solution, depend on these parameters. So, it is possible to control this type of device by manipulating these parameters.

In general, it can be said that the distance from the rotor to the center of mass ( $k_{31}$ in dimensionless form) is a stabilizing parameter that can lead to the bifurcation to conical solutions.

\section{Discussion}

In this paper, the effect of different configuration parameters on stability and stabilization time of pararotors has been presented. Two kinds of stable response were found: straight and conical solutions. The numerical results obtained show a good agreement with the test measurements done in previous works $[12,19]$.

Future investigations should be conducted to explore regions of the $N_{e}^{\prime}, k_{e}$ plane to define the stability regions limits. In-flight tests should also be done in order to check results, to complement the wind tunnel tests. Moreover, future studies should include control strategies and guiding devices. Finally, an experimental effort should be devoted to characterize blade aerodynamics in order to complete the aerodynamic loading model of pararotor blades.

\section{Conflict of interest statement}

None declared.

\section{References}

[1] Z. Shpund, D. Levin, Measurement of the static and dynamic coefficients of a cross-type parachute in subsonic flow, in: 11th AIAA Aerodynamic Decelerator Systems Technology Conference, San Diego, CA, April 9-11, 1991, AIAA Paper 91-0871-CP, pp. 295-303.

[2] D. Levin, Z. Shpund, Dynamic investigation of the angular motion of a rotating body-parachute system, J. Aircr. 32 (1) (1995) 93-99.

[3] Z. Shpund, D. Levin, Forebody influence on rotating parachute aerodynamic properties, J. Aircr. 34 (2) (1997) 181-186.

[4] D. Levin, Z. Shpund, Canopy geometry effect on the aerodynamic behaviour of cross-type parachutes, J. Aircr. 34 (5) (1997) 648-652.

[5] L. Karlsen, D. Borgström, L. Paulsson, Aerodynamics of a rotating body descending from the separation position of an artillery munition shell, in: 11th AIAA Aerodynamic Decelerator Systems Technology Conference, San Diego, CA, April 9-11, 1991, AIAA-91-0870-CP, pp. 288-294.

[6] A. Rosen, D. Seter, Vertical autorotation of a single winged samara, Trans. Am. Soc. Mech. Eng. 58 (Dec. 1991) 1064-1070.

[7] D. Seter, A. Rosen, Stability of the vertical autorotation of a single winged samara, Trans. Am. Soc. Mech. Eng. 59 (Dec. 1992) 1000-1008.

[8] P. Crimi, Analysis of samara-wing decelerator steady-state characteristics, J. Aircr. 25 (1) (Jan. 1988) 41-47.

[9] J. Piechocki, Estudio de la dinámica del vuelo de un decelerador aerodinámico basado en el concepto de pararotor, Tesis doctoral, Universidad Nacional de La Plata, Argentina, March 2011.

[10] V. Nadal Mora, Comportamiento aerodinámico de sondas atmosféricas en entornos aeroportuarios, Tesis doctoral, Universidad Politécnica de Madrid, España, May 2005.

[11] V. Nadal Mora, A. Sanz Andrés, J. Piechocki, S. Pezzotti, Ensayos en túnel aerodinámico de modelos de deceleradores aerodinámicos de alas rotatorias, in: II Congreso Argentino de Ingeniería Aeronáutica, CAIA 2, Córdoba, Argentina, 2010.

[12] J. Piechocki, V. Nadal Mora, A. Sanz Andrés, S. Pezzotti, Modelos teóricos y numéricos para predecir el comportamiento dinámico de pararotores, in: II Congreso Argentino de Ingeniería Aeronáutica, CAIA 2, Córdoba, Argentina, 2010.

[13] J. Piechocki, V. Nadal Mora, A. Sanz Andrés, Estudio teórico del efecto del desplazamiento del plano que contiene a las palas con respecto al centro de masas en la dinámica del vuelo de un desacelerador aerodinámico de alas rotatorias, in: I Congreso Argentino de Ingeniería Mecánica, CAIM, Bahía Blanca, 2008.

[14] A. Sanz-Andres, V. Nadal Mora, J. Piechocki, Pararotors for planetary atmosphere exploration, in: IPPW-5, Burdeos, France, 2007.

[15] V. Nadal Mora, A. Sanz-Andrés, A. Cuerva, Model of the aerodynamic behaviour of a pararotor, J. Aircr. 43 (6) (November-December 2006) 1893-1903.

[16] V. Nadal Mora, A. Sanz-Andres, Stability analysis of a free falling pararotor, J. Aircr. 43 (4) (July-August 2006) 980-986.

[17] J. Piechocki, V. Nadal Mora, A. Sanz Andrés, Pararotor dynamics: center of mass displacement from the blade plane-analytical approach, J. Aircr. 51 (2) (2014) 651-660.

[18] D. Seter, A. Rosen, Dynamics of systems that include wings in autorotation, J. Dyn. Syst. Meas. Control 121 (2) (1999).

[19] A. Kellas, The guided samara: design and development of a controllable singlebladed autorotating vehicle, Master thesis, Massachusetts Institute of Technology, 2007. 
[20] G.E. Dorrington, Concept options for the aerial survey on Titan, Adv. Space Res. 47 (2011).

[21] T.J. Steiner, L.A. Young, Rotary wing decelerator use on Titan, in: IPPW8, Portsmouth, USA, 2011.

[22] L.A. Young, G. Briggs, E. Aiken, G. Pisanich, Rotary-Wing Decelerators for Probe Descent Through the Atmosphere of Venus, DTIC, 2004.

[23] G.R. Spedding, J. McArthur, Span efficiencies of wings at low Reynolds numbers, J. Aircr. 47 (1) (January-February 2010).

[24] J.G. Leishman, Principles of Helicopter Aerodynamics, Cambridge Aerospace Series, Cambridge University Press, Cambridge, UK, 2000.
[25] J. Diebel, Representing Attitude: Euler Angles, Unit Quaternions, and Rotation Vectors, Stanford University, 2006.

[26] Ai Chun Fang, B.G. Zimmerman, Digital simulation of rotational kinematics, NASA TN D-5302, 1969.

[27] J.C. Butcher, Numerical Methods for Ordinary Differential Equations, John Wiley \& Sons, 2003.

[28] V. Nadal Mora, J. Piechocki, A. Sanz Andrés, Experimental research on a vertically falling rotating wing decelerator model, in: 19th AIAA Aerodynamic Decelerator Systems Technology Conference and Seminar, 2007, AIAA 2007-2538. 